\title{
Sarcoplasmic Masses in the Skeletal Muscle of a Stranded Pigmy Sperm Whale (Kogia breviceps)
}

Eva Sierra, ${ }^{1,2}$ Antonio Espinosa de los Monteros, ${ }^{1}$ Antonio Fernández, ${ }^{1}$ Manuel Arbelo, ${ }^{1}$ María José Caballero, ${ }^{1}$ Miguel Rivero, ${ }^{1}$ and Pedro Herráez ${ }^{1}{ }^{1}$ Department of Veterinary Pathology, Institute for Animal Health, Veterinary School, Universidad de Las Palmas de Gran Canaria, Trasmontaña S/N, 35416, Arucas, Las Palmas, Spain; ${ }^{2}$ Corresponding author (email: esierra@ becarios.ulpgc.es)

ABSTRACT: We measured the abundance of sarcoplasmic masses within skeletal muscle myocytes of an adult female stranded pigmy sperm whale (Kogia breviceps). The presence of these masses in other species has been reported in association with myopathies, including myotonic dystrophy, the most frequently related pathology. Other histopathologic muscle changes included a high number of internal nuclei, variations in fiber size and shape, and the predominance of type I fibers.

Key words: Cetacean, internal nuclei, sarcoplasmic masses, type I fibers.

Sarcoplasmic masses (SMs) may be morphologically identified as single or multiple homogeneous areas devoid of myofibrils and located under the sarcolemma and central area of myofibers. The masses show irregular staining with periodic acid-Schiff (PAS) and do not stain with phosphotungstic acid hematoxylin (PTAH). At the ultrastructural level, they contain irregularly oriented myofibrillar components with bundles of myofibrils trapped in disorganized areas (Braga et al., 1995; Banker and Engel, 2004).

Sarcoplasmic masses have been observed in normal equine skeletal muscle (Aleman et al., 2005), whereas in humans, they have been observed always in association with various myopathies, such as limb girdle muscular dystrophy, hypothyroid myopathy, and, most frequently, myotonic dystrophy (Banker and Engel, 2004). In animals, SMs have been described in foals (Hegreberg and Reed, 1990) and Japanese quail (Braga et al., 1995) with myotonic dystrophy and in horses with palatal myositis (Blythe et al., 1983).

We report the presence of abundant $\mathrm{SMs}$ in a 2.85-m adult female pigmy sperm whale (Kogia breviceps). The ani- mal was found stranded and dead on the coast of Fuerteventura, Canary Islands, Spain, in August 2006 and was examined at postmortem examination following standard procedures. The carcass condition was established as fresh to moderate decomposition (code 2-3; Geraci and Lounsbury, 2005). Tissue samples from many organs were collected and fixed in $10 \%$ buffered formalin, embedded in paraffin, sectioned, and stained with $\mathrm{H} \& \mathrm{E}$ for evaluation by light microscopy. Samples of transverse and longitudinally oriented skeletal muscles were taken from the middle portion of the longissimus dorsi, as has been proposed (Tulsi, 1975). Additional histochemical stains applied to sections of skeletal muscle included PAS and PTAH (Prophet et al., 1992).

The slow and fast myosin heavy-chain isoforms, recognizing type I and type II muscle fibers, respectively, were analyzed by immunohistochemistry (Sigma Co., St. Louis, Missouri, USA). Ten images per section $(200 \times$ magnification) were taken, using a digital Altra20 camera (2 MegaPixel complementary metal oxide-semiconductor color camera for light microscopy, Olympus Soft Imaging Solutions GmbH, Münster, Germany). Muscle morphology (cross-sectional area [CSA], lesser diameter and number of fiber types) and the mean and standard error were calculated using the digital software CellA (Olympus Soft Imaging Solutions). The same protocol and stains were performed on skeletal muscle interpreted as normal from a 2.98-m adult male pigmy sperm whale (code 1; Geraci and Lounsbury, 2005). Age of case and control animals were based on biologic and morphometric 
parameters reported for $K$. breviceps (Bloodworth and Odell, 2008).

Additionally, skeletal muscle samples were immunostained to detect dystrophin (mouse anti-human monoclonal; Sigma) and $\alpha$-actin (HHF35; mouse anti-human monoclonal; Enzo Biochemical, New York, New York, USA). Mutations in the gene codifying these proteins are associated with a wide range of muscular diseases.

For ultrastructural studies, skeletal muscle samples were fixed in $2.5 \%$ glutaraldehyde in $0.1 \mathrm{M}$ sodium cacodilate buffer ( $\mathrm{pH}$ 7.2). The specimens were postfixed in $1 \%$ osmium tetroxide in $0.2 \%$ veronal buffer, gradually dehydrated in an alcohol series, and embedded in EMbed 812 epoxy resin (Electron Microscopic Science, Hatfield, Pennsylvania, USA). Thin sections were stained with uranyl acetate and lead citrate. The sections were examined with a ZEISS EM-912 transmission electron microscope (Carl Zeiss, Oberkochen, Germany).

Grossly, the animal showed intense multiorganic parasitic infections, morphologically identified as larval cestodes (Phyllobothrium sp.) within the subcutaneous tissues, nematodes (Crassicauda sp.) in muscle connective tissues, Anisakis sp. in stomachs, and unidentified trematodes in liver and pancreas (Dailey, 2001; Aytemizla et al., 2012). Severe emaciation was evident from protuberance of axial bones. Skeletal muscle histopathology revealed abundant SMs (57.8 fibers affected per field at $200 \times$ magnification). Most SMs were found in the periphery of the affected fibers (Fig. 1A), fewer were centrally located (Fig. 1A., left inset), and some encircled the periphery of the myofibers. The SMs were negative for PAS and PTAH stains, indicating the absence of glycogen and myofibrils, respectively.

A variety of morphologic changes were observed, including internalized nuclei associated with an increase in the number of nuclei (nuclear rowing) and variation in fiber size and shape (Fig. 1B). Internal nuclei were seen in both type I and II fibers and affected three morphologically distinct fibers: fibers of normal architecture, fibers altered by incomplete division, and small fibers. Rows of more than 10-12 internally positioned nuclei were more often seen in longitudinal sections compared with transverse sections (Fig. 1B, left inset). Multifocal variation in size and shape, including irregular atrophy of fibers and compensatory hypertrophy, often accompanied by fiber splitting, were frequently observed. Less frequent findings included intranuclear and intrasarcoplasmic vacuolization, segmental myonecrosis, and an increase in the interstitial fibrous stroma, especially in the periphery of the fascicles.

Morphometric data from the case and control animal are summarized in Table 1. The case showed decreased CSA, lesser diameter for type I and II fibers, and a marked decreased in the number of type II fibers, with a higher proportion of type I fibers. Immunohistochemical analysis (Fig. 1C) confirmed the muscular atrophy and predominance of type I fibers and revealed the presence of concentrated deposits of muscle actin within the periphery of some affected fibers (Fig. 1C, left inset), and a diffuse, slightly cytoplasmic, immunostaining for muscle actin was observed in normal and other affected fibers. There was a generalized absence of dystrophin immunostaining in all skeletal muscle samples examined, including the control. These results could be interpreted as an absence of reaction to this commercial antibody. The same stains on skeletal muscle of the control animal revealed no significant change (Fig. 1A-C, right inset). Ultrastructural study revealed that sarcoplasmic masses were not membrane bound and were either devoid of myofilaments or characterized by the presence of sparse myofilaments and irregularly oriented and disorganized myofibrillar components with mitochondria, dilated tubules, and lipofuscin (Fig. 1D). 

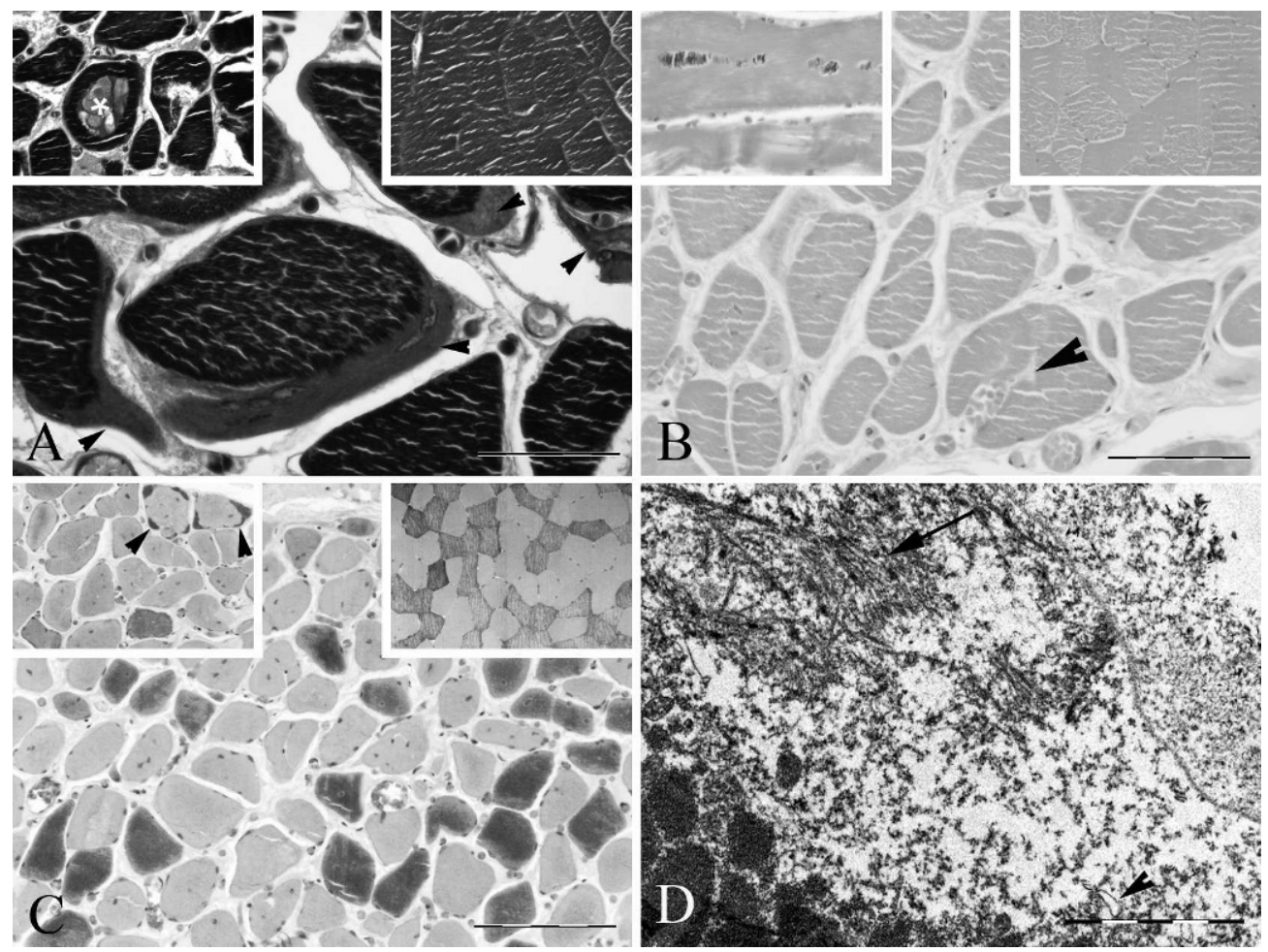

Figure 1. Microscopic features of a pigmy sperm whale (Kogia breviceps) found stranded and dead on the coast of Fuerteventura, Canary Islands, Spain, August 2006 (case). Comparisons are to an adult male pigmy sperm whale (control). (A) Cross section from the skeletal muscle of the case animal. Note high number of peripheral sarcoplasmic masses (SMs), negatively stained with phosphotungstic acid hematoxylin (PTAH). Inset left: centrally located SMs (PTAH; case animal). Inset right: cross section from control animal. Note absence of subsarcolemmal peripheral masses (PTAH. Bar $=20 \mu \mathrm{m}$ ). (B) Cross section from the skeletal muscle of case animal. Note morphologic changes, including internalized nuclei, variation in fiber size and shape; and fiber splitting (arrowhead). H\&E. Inset left: longitudinal section of case animal. Fiber presenting rows of high number of internally positioned nuclei (nuclear rowing). HE. Inset right: cross section from control animal. H\&E. Bar $=20 \mu \mathrm{m}$. (C) Cross section from skeletal muscle of case animal. High proportion of type I fibers conducting to a deviation to the normal mosaic-like arrangement of fibers. Immunohistochemistry for fast-twitch fibers (type II fibers). Inset left: immunohistochemistry for $\alpha$-actin. Cross section from case animal. Some fibers express a peripheral condensation of the $\alpha$-actin protein. Bar $=50 \mu$ m. Inset right: cross section from control animal. Normal distribution pattern of muscle fiber types observed in an adult male pigmy sperm whale. Immunohistochemistry for fast-twitch fibers (type II fibers). Bar $=50 \mu \mathrm{m}$. (D) Electron micrograph. The subsarcolemmal mass contained bundles of disorganized myofibrils (arrows), destruction of myofibrillar components, and dilated sarcoplasmic reticulum and tubules (arrowhead). Bar=2 $\mu \mathrm{m}$.

Pathologic changes observed in the pigmy sperm whale were restricted to the skeletal muscle and mainly included the presence of SMs, changes in the number and location of sarcolemmal nuclei, fiber size and shape variations, cytoarchitectural changes, and alterations in fiber type proportion. All these changes have been described in association with many muscle diseases and in aging muscle
(Åström and Adams, 1982; Valentine and McGawin, 2007), but they are also consistent with many of the histologic criteria established for the diagnosis of muscular dystrophies in humans and animals (Åström and Adams, 1982; Hegreberg and Reed, 1990; Sarli et al., 1994).

Muscular dystrophies are a group of genetic disorders that mostly comprise loss of function mutations or deletions in 
TABLE 1. Comparison of morphologic characteristics of longissimus dorsi muscle fibers from pigmy sperm whale (Kogia breviceps) diagnosed with sarcoplasmic masses, compared to a control pigmy sperm whale. Values are reported as mean \pm SD.

\begin{tabular}{lccccc}
\hline & \multicolumn{2}{c}{ Control } & & \multicolumn{2}{c}{ Case } \\
\cline { 2 - 3 } \cline { 5 - 6 } & Type I fibers & Type II fibers & & Type I fibers & Type II fibers \\
\hline Cross-sectional area $\left(\mu \mathrm{m}^{2}\right)$ & $797.35 \pm 210.89$ & $686.22 \pm 218.34$ & & $687.92 \pm 360.28$ & $534.45 \pm 229.29$ \\
Lesser diameter $(\mu \mathrm{m})$ & $24.60 \pm 4.68$ & $21.01 \pm 4.89$ & & $21.25 \pm 6.9$ & $18.08 \pm 5.03$ \\
No. & 403 & 385 & & 405 & 186 \\
\hline
\end{tabular}

genes that encode proteins involved in maintaining the structural integrity of the muscle sarcolemmal membrane, in particular those that comprise the dystrophinassociated glycoprotein complex (Gordon and Hoffman, 2001). Identification of cytoskeletal protein defects relies, in part, on immunohistochemical preparations, in which tissue controls are crucial to discriminate pathologic from natural changes. Although there is no evidence on the reactivity of the dystrophin in cetaceans, the reactivity of muscle actin has been described in bottlenose dolphins (Tursiops truncatus), showing a uniformly strong cytoplasmic diffuse immunostaining in myoctyes (Kumar and Cowan, 1994).

Actin accumulation and fiber type disproportion has been described within the main skeletal lesions associated with muscle $\alpha$-actin gene mutations (Laing et al., 2004), which cause a range of pathologically defined congenital myopathies characterized by weakness and pathologic changes of skeletal muscles. Even if the underlying protein defect could not be established definitively, the major histopathologic lesions observed in the muscle of this pigmy sperm whale were comparable to those that have been classically described as typical of myotonic dystrophy in humans and animals, such as SMs, multiple internal nuclei, and fiber type disproportion, especially when two or more changes are seen in combination (Nakamura et al., 1994; Sarli et al., 1994).

This study was supported by the Ministerio de Ciencia, Investigación e Innovación (CGL2009-13052), Spain.

\section{LITERATURE CITED}

Aleman M, Lecouteur RA, Nieto JE, Williams DC, Stanke NJ, Shelton GD. 2005. Sarcoplasmic masses in equine skeletal muscle. Neuromuscul Disord 15:147-153.

Åström KE, Adams RD. 1982. Myotonic disorders. In: Skeletal muscle pathology, Mastalgia FL and Walton J, editors. Walton and Churchill Livingston, New York, New York, pp. 266-286.

Aytemizla I, Dede A, Danyer E, Tonay AM. 2012. Morphological identification of parasites found in the stomach contents of bycaught striped dolphins (Stenella coeruleoalba) from Turkish eastern Mediterranean sea coast. I Black Seal Mediterr Environ 18:238-245.

Banker BQ, Engel AG. 2004. Basic reactions of muscle. In: Myology: Basic and clinical, 3rd Ed., Engel A and Franzini-Armstrong C, editors. McGraw-Hill, New York, New York, pp. 691747.

Bloodworth BE, Odell DK. 2008. Kogia breviceps (Cetacea: Kogiidae). Mamm Species 819:1-12.

Blythe LL, Cardinet GH. 3rd, Meagher DM, Brown MP, Wheat JD. 1983. Palatal myositis in horses with dorsal displacement of the soft palate. $\mathrm{J} \mathrm{Am}$ Vet Med Assoc 183:781-785.

Braga IS. 3rd, Tanaka S, Kimura T, Itakura C, Mizutani M. 1995. Inherited muscular disorder in mutant Japanese quail (Coturnix coturnix japonica): Relationship between the development of muscle lesions and age. J Comp Pathol 113:131-143.

Dailey MD. 2001. Parasitic diseases. In: CRC handbook of marine mammal medicine, 2nd Ed., Dierauf AL and Gulland FMD, editors. CRC Press, Boca Raton, Florida, pp. 361367.

Geraci JR, Lounsbury VJ. 2005. Specimen and data collection. In: Marine mammals ashore: A field guide for strandings, 2nd Ed., Geraci JR and Lounsbury VL, editors. Texas A\&M University Sea Grant College Program, Galveston, Texas, pp. 182-184.

Gordon ES, Hoffman EP. 2001. The abc's of limbgirdle muscular dystrophy: Alpha-sarcoglycanopathy, bethlem myopathy, calpainopathy and more. Curr Opin Neurol 14:567-573. 
Hegreberg GA, Reed SM. 1990. Skeletal muscle changes associated with equine myotonic dystrophy. Acta Neuropathol 80:426-431.

Kumar D, Cowan DF. 1994. Cross-reactivity of antibodies to human antigens with tissues of the bottlenose dolphin, Tursiops truncatus, using immunoperoxidase techniques. Mar Mamm Sci 10:188-194.

Laing NG, Clarke NF, Dye DE, Liyanage K, Walker KR, Kobayashi Y, Shimakawa S, Hagiwara T, Ouvrier R, Sparrow JC, et al. 2004. Actin mutations are one cause of congenital fibre type disproportion. Ann Neurol 56:689-694.

Nakamura N, Doi T, Furuoka H, Katoh M, Inada I, Iguchi H, Osame S, Matsui T. 1994. Muscular dystrophy of the diaphragmatic muscles in Holstein-Friesian cows. J Vet Med Sci 56:993-994.
Prophet EB, Mills B, Arrington JB and Sobin LH, editors. Laboratory methods in histotechnology (Armed Forces Institute of Pathology). American Registry of Pathology, Washington, DC, pp. 5358, 98-100, 149-160, 197-198.

Sarli G, Della Salda L, Marcato PS. 1994. Dystrophylike myopathy in a foal. Vet Rec 135:156-60.

Tulsi RS. 1975. Observations on the structure of the dorsal muscle in the bottle-nose dolphin (Tursiops truncatus). J Anat 119:39-48.

Valentine BA, McGawin MD. 2007. Skeletal muscle. In: Pathologic basis of veterinary disease, 4th Ed. Mosby Elsevier, St Louis, Missouri, pp. 9891039.

Submitted for publication 9 April 2012. Accepted 30 January 2013. 